\title{
Cooperative Approaches in Introducing Undergraduates to Protein Crystallography
}

Krystle J. McLaughlin*

Department of Biological Sciences, Lehigh University, 111 Research Dr., Bethlehem PA 18015

*Email: krm514@lehigh.edu

Undergraduates in the biological sciences regularly see protein structures, usually solved by x-ray crystallography, but often have not been exposed to the origin of the structures in their education, or have the tools to interpret these structures. Here I will discuss activities to introduce students to protein x-ray crystallography in a lab setting, in the absence of an on-site diffractometer. In this cooperative multi-activity module, students learn basic protein crystallography lab skills, learn to navigate online databases, such as the RCSB Protein Data Bank, and additionally examine physical models of protein structure. They are introduced to the basics of model building and visualization through Coot and PyMOL, learning to obtain, use and interpret electron density maps with structure. 